\title{
Group Classification of a General Bond-Option Pricing Equation of Mathematical Finance
}

\author{
Tanki Motsepa, ${ }^{1}$ Chaudry Masood Khalique, ${ }^{1}$ and Motlatsi Molati ${ }^{1,2}$ \\ ${ }^{1}$ International Institute for Symmetry Analysis and Mathematical Modelling, Department of Mathematical Sciences, \\ North-West University, Mafikeng Campus, Private Bag X 2046, Mmabatho 2735, South Africa \\ ${ }^{2}$ Department of Mathematics and Computer Science, University of Lesotho, Roma 180, Lesotho
}

Correspondence should be addressed to Chaudry Masood Khalique; masood.khalique@nwu.ac.za

Received 30 January 2014; Accepted 24 March 2014; Published 13 April 2014

Academic Editor: Imran Naeem

Copyright (C) 2014 Tanki Motsepa et al. This is an open access article distributed under the Creative Commons Attribution License, which permits unrestricted use, distribution, and reproduction in any medium, provided the original work is properly cited.

\begin{abstract}
We carry out group classification of a general bond-option pricing equation. We show that the equation admits a three-dimensional equivalence Lie algebra. We also show that some of the values of the constants which result from group classification give us wellknown models in mathematics of finance such as Black-Scholes, Vasicek, and Cox-Ingersoll-Ross. For all such values of these arbitrary constants we obtain Lie point symmetries. Symmetry reductions are then obtained and group invariant solutions are constructed for some cases.
\end{abstract}

\section{Introduction}

The theory of option pricing began in 1900 when the French mathematician Bachelier [1] deduced an option pricing formula based on the assumption that stock prices follow a Brownian motion. The Black-Scholes equation

$$
u_{t}+\frac{1}{2} x^{2} \sigma^{2} u_{x x}+r\left(x u_{x}-u\right)=0
$$

was introduced by Black and Scholes [2] as the general equilibrium theory of option pricing which is particularly attractive because the final formula is a function of observable variables. Merton [3] extended the Black-Scholes theory of option pricing by introducing more assumptions and found new explicit formulas for pricing both the call and put options as well as the warrants and the down-and-out options. The equation is mainly used to find the fair price of a financial instrument (option or derivative) and to find the implied volatility.

The first bond pricing equation

$$
u_{t}+\frac{1}{2} \sigma^{2} u_{x x}+\kappa(\theta-x) u_{x}-x u=0
$$

was introduced by Vasicek [4] and thereafter many other researchers (see, e.g., [5-12]) came up with other one-factor models which modelled the term structure of interest rates.

Many differential equations, including financial mathematics equations, involve parameters, arbitrary elements, or functions, which need to be determined. Usually, these arbitrary parameters are determined experimentally. However, the Lie symmetry approach through the method of group classification has proven to be a versatile tool in specifying the forms of these parameters systematically (see, e.g., [13-20]).

In 1881, Lie [21] was the first one to investigate the problem of group classification. In this regard, he studied a linear second-order partial differential equation with two independent variables. Suppose a differential equation contains an arbitrary element $f(u)$. The main idea of group classification of this differential equation is to find the Lie point symmetries of the differential equation with arbitrary element $f(u)$ and then find all possible forms of $f(u)$ for which the principal Lie algebra can be extended.

Semi-invariants for the $(1+1)$ linear parabolic equations with two independent variables and one dependent variable were derived by Johnpillai and Mahomed [22]. In addition, joint invariant equation was obtained for the linear parabolic equation and the $(1+1)$ linear parabolic equation was 
reducible via a local equivalence transformation to the onedimensional heat equation. In [23], a necessary and sufficient condition for the parabolic equation to be reducible to the classical heat equation under the equivalence group was provided which improved the work done in [22].

Goard [24] found group invariant solutions of the bond pricing equation by the use of classical Lie method. The solutions obtained were shown to satisfy the condition for the bond price, that is, $P(r, T)=1$, where $P$ is the price of the bond. Here $r$ is the short-term interest rate which is governed by the stochastic differential equation and $T$ is time to maturity.

In [25], the fundamental solutions were obtained for a number of zero-coupon bond models by transforming the one-factor bond pricing equations corresponding to the bond models to the one-dimensional heat equation whose fundamental solution is well known. Subsequently, the transformations were used to construct the fundamental solutions for zero-coupon bond pricing equations.

Sinkala et al. [26] computed the zero-coupon bonds (group invariant solutions satisfying the terminal condition $u(T, T)=1$ ) using symmetry analysis for the Vasicek and Cox-Ingersoll-Ross (CIR) equations, respectively. In [27] an optimal system of one-dimensional subalgebras was derived and used to construct distinct families of special closed-form solutions of CIR equation. In [20], group classification of the linear second-order parabolic partial differential equation

$$
u_{t}+\frac{1}{2} \rho^{2} x^{2 \gamma} u_{x x}+\left(\alpha+\beta x-\lambda \rho x^{\gamma}\right) u_{x}-x u=0,
$$

where $\alpha, \beta, \lambda, \rho$, and $\gamma$ are constants, was carried out. Lie point symmetries and group invariant solutions were found for certain values of $\gamma$. Also the forms where equation (3) admitted the maximal seven Lie point symmetry algebra were transformed into the heat equation. Vasicek, CIR, and Longstaff models were recovered from group classification and some other equations were derived which had not been considered before in the literature. Furthermore, Mahomed et al. [28] used the invariant conditions developed in [23] to carry out group classification of [20] and some new cases were discovered.

Dimas et al. [29] investigated some of the well-known equations that arise in mathematics of finance, such as BlackScholes, Longtsaff, Vasicek, CIR, and heat equations. Lie point symmetries of these equations were found and their algebras were compared with those of the heat equation. The equations with seven symmetries were transformed to the heat equation.

In this paper, we study a general bond-option pricing equation. The partial differential equation which will be investigated is a generalisation of (1) and (2) and is given by

$$
u_{t}+\alpha x^{p} u_{x x}+\lambda(\beta-x) u_{x}+\gamma x^{q} u=0,
$$

where $t$ is time, $x$ is the stock (share or equity) price or instantaneous short-term interest rate at current time $t$, and $u(t, x)$ is the current value of the option or bond. Here $p \geq 0$, $q \geq 0$, and $\alpha, \gamma, \lambda$, and $\beta$ are constants with $\alpha, \lambda, \gamma \neq 0$. When $q=0$, (4) is the option pricing equation and it is the bond pricing $\operatorname{PDE}$ when $q=1$.
This paper is structured as follows. In Section 2, we find two classifying equations on which group classification of (4) depends, one for $p \neq 2$ and the other for $p=2$. Then we use the two equations to find possible values for arbitrary constants for which (4) admits nontrivial Lie point symmetry algebras. In Section 3, we obtain symmetry reductions and construct group-invariant solutions for Case 2.1(1) and finally in Section 4 we give conclusions.

\section{Determination of Classifying Equations of (4)}

The Lie point symmetries for (4) are given by the vector field:

$$
X=\tau(t, x, u) \frac{\partial}{\partial t}+\xi(t, x, u) \frac{\partial}{\partial x}+\eta(t, x, u) \frac{\partial}{\partial u}
$$

if and only if

$$
\left.X^{[2]}\left(u_{t}+\alpha x^{p} u_{x x}+\lambda(\beta-x) u_{x}+\gamma x^{q} u\right)\right|_{(4)}=0,
$$

where $X^{[2]}$ is the second prolongation of $X$ defined as

$$
X^{[2]}=X+\zeta_{1} \frac{\partial}{\partial u_{t}}+\zeta_{2} \frac{\partial}{\partial u_{x}}+\zeta_{22} \frac{\partial}{\partial u_{x x}}
$$

Here $\zeta_{i}$ 's are given by

$$
\begin{aligned}
& \zeta_{1}=D_{t}(\eta)-u_{t} D_{t}(\tau)-u_{x} D_{t}(\xi), \\
& \zeta_{2}=D_{x}(\eta)-u_{t} D_{x}(\tau)-u_{x} D_{x}(\xi), \\
& \zeta_{22}=D_{x}\left(\zeta_{2}\right)-u_{t x} D_{x}(\tau)-u_{x x} D_{x}(\xi),
\end{aligned}
$$

where the total derivatives $D_{t}$ and $D_{x}$ are defined as

$$
\begin{aligned}
& D_{t}=\frac{\partial}{\partial t}+u_{t} \frac{\partial}{\partial u}+u_{t x} \frac{\partial}{\partial u_{x}}+u_{t t} \frac{\partial}{\partial u_{t}}+\cdots \\
& D_{x}=\frac{\partial}{\partial x}+u_{x} \frac{\partial}{\partial u}+u_{x x} \frac{\partial}{\partial u_{x}}+u_{t x} \frac{\partial}{\partial u_{t}}+\cdots
\end{aligned}
$$

To perform the group classification of (4) it turns out that we need to consider two cases of $p$ separately: $p \neq 2$ and $p=2$.

2.1. Classifying Equation of (4) for $p \neq 2$. Expanding the determining equation (6), we obtain

$$
\begin{array}{r}
\gamma \xi q u x^{q-1}-\gamma u x^{q} \eta_{u}+\gamma u x^{q} \xi_{u} u_{x}+\gamma \eta x^{q}+\eta_{t}-\xi_{t} u_{x} \\
-\lambda \xi u_{x}+\beta \lambda \eta_{x}-\lambda x \eta_{x}+\gamma u x^{q} \tau_{t}-\gamma^{2} u^{2} x^{2 q} \tau_{u} \\
+\gamma \lambda u x^{q+1} \tau_{u} u_{x}+\beta \lambda \tau_{t} u_{x}-\lambda x \tau_{t} u_{x}-\beta \lambda u_{x} \xi_{x}
\end{array}
$$




$$
\begin{aligned}
& +\lambda x u_{x} \xi_{x}-\gamma \lambda u x^{q+1} \tau_{x}-\beta \gamma \lambda u x^{q} \tau_{u} u_{x}+\beta \gamma \lambda u x^{q} \tau_{x} \\
& +\lambda^{2} x^{2} u_{x} \tau_{x}+\beta^{2} \lambda^{2} u_{x} \tau_{x}-2 \beta \lambda^{2} x u_{x} \tau_{x} \\
& -2 \alpha x^{p} \tau_{u} u_{x} u_{t x}-2 \alpha x^{p} \tau_{x} u_{t x}+\alpha \xi p x^{p-1} u_{x x} \\
& -2 \alpha x^{p} \xi_{u} u_{x} u_{x x}-2 \alpha x^{p} \xi_{x} u_{x x}+x^{p} \\
& \times\left(\alpha \tau_{t} u_{x x}-\alpha \gamma u \tau_{u} u_{x x} x^{q}-\alpha \lambda x \tau_{x} u_{x x}\right. \\
& \left.\quad+\alpha \beta \lambda \tau_{x} u_{x x}+\alpha u_{x}^{2} \eta_{u u}+2 \alpha u_{x} \eta_{x u}\right) \\
& +\alpha \gamma u u_{x}^{2} \tau_{u u} x^{p+q}-\alpha \lambda x^{p+1} u_{x}^{3} \tau_{u u}-\alpha x^{p} u_{x}^{3} \xi_{u u} \\
& -2 \alpha x^{p} u_{x}^{2} \xi_{x u}-\alpha x^{p} u_{x} \xi_{x x}+\alpha x^{p} \eta_{x x} \\
& +2 \alpha \gamma u u_{x} x^{p+q} \tau_{x u}+\alpha^{2} x^{2 p} u_{x}^{2} \tau_{u u} u_{x x}-2 \alpha \lambda x^{p+1} u_{x}^{2} \tau_{x u} \\
& +\alpha \beta \lambda x^{p}\left(u_{x}^{3} \tau_{u u}+2 u_{x}^{2} \tau_{x u}\right)+\alpha \gamma u \tau_{x x} x^{p+q} \\
& +\alpha x^{2 p} u_{x x}\left(2 u_{x} \tau_{x u}+\tau_{x x}\right)-\alpha \lambda x^{p+1} u_{x} \tau_{x x} \\
& +\alpha \beta \lambda x^{p} u_{x} \tau_{x x}=0 .
\end{aligned}
$$

Separating (10) with respect to the derivatives of $u$, since the functions $\tau, \xi$, and $\eta$ do not depend on them, leads to the following linear PDEs:

$$
\begin{gathered}
\tau_{u}=0, \\
\xi_{u}=0, \\
\eta_{u u}=0, \\
\tau_{x}=0, \\
p \xi+x\left(\tau_{t}-2 \xi_{x}\right)=0, \\
u \gamma x^{q} \eta_{u}+x \lambda \eta_{x}-q u \gamma x^{q-1} \xi-\gamma x^{q} \eta-\alpha x^{p} \eta_{x x} \\
-u \gamma x^{q} \tau_{t}-\eta_{t}-\beta \lambda \eta_{x}=0, \\
\lambda \xi+\alpha x^{p} \xi_{x x}+x \lambda \tau_{t}+\xi_{t}+\lambda(\beta-x) \xi_{x} \\
-2 \alpha x^{p} \eta_{x u}-\beta \lambda \tau_{t}=0 .
\end{gathered}
$$

To solve the above system of equations, we first observe from (11) and (14) that $\tau$ does not depend on $x$ and $u$, which means that $\tau$ is a function of $t$. Thus

$$
\tau=\tau(t)
$$

Equation (12) implies that $\xi$ depends on both $t$ and $x$ but not on $u$. Hence

$$
\xi=\xi(t, x)
$$

Integration of (13) with respect to $u$ twice gives

$$
\eta(t, x, u)=A(t, x) u+B(t, x)
$$

where $A(t, x)$ and $B(t, x)$ are arbitrary functions of $t$ and $x$. Using the expressions for $\tau$ and $\xi$ in (15) and integrating with respect to $x$ lead to

$$
\xi(t, x)=c(t) x^{p / 2}-\frac{x \tau^{\prime}(t)}{p-2}, \quad p \neq 2,
$$

where $c(t)$ is an arbitrary function of $t$. Using (18), (20), and (21) in (17) yields

$$
\begin{aligned}
8 \alpha x^{p+1} & A_{x}(t, x)(2-p)+c(t) \alpha p x^{-1+3 p / 2}(p-2)^{2} \\
& -2 c(t) \lambda x^{1+p / 2}(p-2)^{2}-4 x^{2} \tau^{\prime \prime}(t) \\
& +2 \beta \lambda p C(t) x^{p / 2}(p-2)+4 x^{p / 2} x c^{\prime}(t)(p-2) \\
& +\lambda\left(4 x^{2}(p-2)+4 \beta x(1-p)\right) \tau^{\prime}(t) \\
= & 0 .
\end{aligned}
$$

Integrating (22) with respect to $x$ and solving for $A$ we obtain

$$
\begin{aligned}
A(t, x)= & d(t)+\frac{x^{-p-1}}{4 \alpha(p-2)^{2}} \\
& \times\left\{(p-2)^{2} x^{p / 2} c(t)\left(p \alpha x^{p}+2 \lambda x(x-\beta)\right)\right. \\
& \left.\quad-2 x^{3} \tau_{t t}\right\}-\frac{x^{-p-1}}{4 \alpha(p-2)^{2}} \\
& \times\left\{2 x ^ { 2 } \left(2(p-2) x^{p / 2} c^{\prime}(t)\right.\right. \\
& \left.\left.\quad+(2-p) \lambda \tau^{\prime}(t)(\beta-x)\right)\right\},
\end{aligned}
$$

where $d(t)$ is an arbitrary function of $t$.

Using (18)-(21) and (23) in (16) gives

$$
\begin{aligned}
& 16 \alpha(p-2)^{2} x^{2+p / 2}\left(\alpha x^{p} B_{x x}+B \gamma x^{q}+B_{t}+\beta \lambda B_{x}-\lambda x B_{x}\right) \\
& +8 u \alpha(p-2) x^{2+p / 2}\left(2 \gamma(p-q-2) x^{q}\right. \\
& -\lambda(p-2)(p-1)) \tau_{t} \\
& +8 u \lambda^{2}(p-2)^{2} x^{4-p / 2} \tau_{t}+8 \beta \lambda u(p-2) \\
& \times\left\{\alpha(p-1) p x^{1+p / 2} \tau_{t}-\beta \lambda(p-1) x^{2-p / 2} \tau_{t}\right. \\
& \left.+\lambda(2 p-3) x^{3-p / 2} \tau_{t}\right\} \\
& +u\left\{8 ( p - 2 ) \left(2 \alpha(p-2) d_{t} x^{2+p / 2}+\alpha(p-1) x^{2+p / 2} \tau_{t t}\right.\right. \\
& \left.\left.\quad-2 x^{3} c_{t t}\right)+8 x^{4-p / 2} \tau_{t t t}\right\} \\
& +\alpha(p-2)^{2} c(t) x^{p-1} u\left\{\alpha p^{3} x^{p}-6 \alpha p^{2} x^{p}+8 \alpha p x^{p}\right. \\
& \left.\quad-8 \beta \lambda p x+16 \gamma q x^{q+2}\right\} \\
& +4 \lambda^{2}(p-2)^{2} x u c(t)(x-\beta)(-\beta p+p x-2 x) \\
& =0 .
\end{aligned}
$$


Since the functions $B, c, d$, and $\tau$ do not depend on $u$, we split (24) with respect to $u$ and get

$$
\begin{gathered}
1: B_{t}+\alpha x^{p} B_{x x}+\lambda(\beta-x) B_{x}+\gamma x^{q} B=0 \\
u: 8 \alpha(p-2) x^{2+p / 2}\left(2 \gamma(p-q-2) x^{q}\right. \\
-\lambda(p-2)(p-1)) \tau_{t} \\
-8 \lambda^{2}(p-2)^{2} x^{4-p / 2} \tau_{t}+8 \beta \lambda(p-2) \\
\times\left(\alpha(p-1) p x^{1+p / 2} \tau_{t}-\beta \lambda(p-1) x^{2-p / 2} \tau_{t}\right. \\
\left.+\lambda(2 p-3) x^{3-p / 2} \tau_{t}\right) \\
+\left(8 ( p - 2 ) \left(2 \alpha(p-2) d_{t} x^{2+p / 2}+\alpha(p-1) x^{2+p / 2} \tau_{t t}\right.\right. \\
\left.\left.\quad-2 x^{3} c_{t t}\right)+8 x^{4-p / 2} \tau_{t t t}\right) \\
+\alpha(p-2)^{2} c(t) x^{p-1}\left(\alpha p^{3} x^{p}-6 \alpha p^{2} x^{p}+8 \alpha p x^{p}\right. \\
\left.\quad-8 \beta \lambda p x+16 \gamma q x^{q+2}\right) \\
+4 \lambda^{2}(p-2)^{2} x c(t)(x-\beta)(-\beta p+p x-2 x) \\
=0
\end{gathered}
$$

It is clear that $B$ satisfies the original equation of (4). Rewriting (26) yields our classifying equation as

$$
\begin{aligned}
& h_{0}(t) x^{5 p / 2}+h_{1}(t) x^{q+2+3 p / 2}+h_{2}(t) x^{1+3 p / 2}+h_{3}(t) x^{4} \\
& \quad+h_{4}(t) x^{3}+h_{5}(t) x^{p+2}+h_{6}(t) x^{p+q+3}+h_{7}(t) x^{p+3} \\
& \quad+h_{8}(t) x^{3+p / 2}+h_{9}(t) x^{2+p / 2}+h_{10}(t) x^{4+p / 2}+h_{11}(t) x^{5} \\
& =0
\end{aligned}
$$

where

$$
\begin{gathered}
h_{0}(t)=\alpha^{2}(p-4)(p-2)^{3} p c(t), \\
h_{1}(t)=16 \alpha \gamma(p-2)^{2} q c(t), \\
h_{2}(t)=-8 \alpha \beta \lambda(p-2)^{2} p c(t), \\
h_{3}(t)=8 \beta \lambda^{2}(p-2)(2 p-3) \tau^{\prime}(t), \\
h_{4}(t)=-8 \beta^{2} \lambda^{2}(p-2)(p-1) \tau^{\prime}(t), \\
h_{5}(t)=8 \alpha \beta \lambda(p-2)(p-1) p \tau^{\prime}(t), \\
h_{6}(t)=16 \alpha \gamma(p-2)(p-q-2) \tau^{\prime}(t), \\
h_{7}(t)=8 \alpha(p-2)\left((p-1) \tau^{\prime \prime}(t)-\lambda(p-2)\right. \\
\left.\times(p-1) \tau^{\prime}(t)+2(p-2) d^{\prime}(t)\right),
\end{gathered}
$$

$$
\begin{gathered}
h_{8}(t)=-8 \beta \lambda^{2}(p-2)^{2}(p-1) c(t), \\
h_{9}(t)=4 \beta^{2} \lambda^{2}(p-2)^{2} p c(t), \\
h_{10}(t)=4(p-2)\left(\lambda^{2} p^{2} c(t)-4 \lambda^{2} p c(t)\right. \\
\left.+4 \lambda^{2} c(t)-4 c^{\prime \prime}(t)\right), \\
h_{11}(t)=-8\left(\lambda^{2} p^{2} \tau^{\prime}(t)-4 \lambda^{2} p \tau^{\prime}(t)\right. \\
\left.+4 \lambda^{2} \tau^{\prime}(t)-\tau^{\prime \prime \prime}(t)\right) .
\end{gathered}
$$

2.2. Classifying Equation of (4) for $p=2$. In the case when $p=2$ in (4), we proceed as above to obtain the determining equation as

$$
\begin{aligned}
q u \gamma \xi & x^{q-1}+\gamma \eta x^{q}+u \gamma \tau_{t} x^{q}-u \beta \gamma \lambda u_{x} \tau_{u} x^{q}+u \beta \gamma \lambda \tau_{x} x^{q} \\
& +u \gamma u_{x} \xi_{u} x^{q}-u \gamma \eta_{u} x^{q}-u^{2} \gamma^{2} \tau_{u} x^{2 q}+u \gamma \lambda u_{x} \tau_{u} x^{q+1} \\
& -u \gamma \lambda \tau_{x} x^{q+1}-u \alpha \gamma \tau_{u} u_{x x} x^{q+2}+u \alpha \gamma u_{x}^{2} \tau_{u, u} x^{q+2} \\
& +2 u \alpha \gamma u_{x} \tau_{x u} x^{q+2}+u \alpha \gamma \tau_{x x} x^{q+2}+\alpha^{2} u_{x}^{2} u_{x x} \tau_{u u} x^{4} \\
& +2 \alpha^{2} u_{x} u_{x x} \tau_{x u} x^{4}+\alpha^{2} u_{x x} \tau_{x x} x^{4}-\alpha \lambda \tau_{x} u_{x x} x^{3} \\
& -\alpha \lambda u_{x}^{3} \tau_{u u} x^{3}-2 \alpha \lambda u_{x}^{2} \tau_{x u} x^{3}-\alpha \lambda u_{x} \tau_{x x} x^{3} \\
& +\lambda^{2} u_{x} \tau_{x} x^{2}-2 \alpha u_{x} \tau_{u} u_{t x} x^{2}-2 \alpha \tau_{x} u_{t x} x^{2}+\alpha \tau_{t} u_{x x} x^{2} \\
& +\alpha \beta \lambda \tau_{x} u_{x x} x^{2}-2 \alpha u_{x} \xi_{u} u_{x x} x^{2}-2 \alpha \xi_{x} u_{x x} x^{2} \\
& +\alpha \beta \lambda_{x}^{3} \tau_{u u} x^{2}+2 \alpha \beta \lambda u_{x}^{2} \tau_{x u} x^{2}+\alpha \beta \lambda u_{x} \tau_{x x} x^{2} \\
& -\alpha u_{x}^{3} \xi_{u u} x^{2}-2 \alpha u_{x}^{2} \xi_{x u} x^{2}-\alpha u_{x} \xi_{x x} x^{2}+\alpha u_{x}^{2} \eta_{u u} x^{2} \\
& +2 \alpha u_{x} \eta_{x u} x^{2}+\alpha \eta_{x x} x^{2}-\lambda u_{x} \tau_{t} x-2 \beta \lambda^{2} u_{x} \tau_{x} x \\
& +\lambda u_{x} \xi_{x} x-\lambda \eta_{x} x+2 \alpha \xi u_{x x} x-\lambda u_{x} \xi+\beta \lambda u_{x} \tau_{t} \\
+ & \beta^{2} \lambda^{2} u_{x} \tau_{x}-u_{x} \xi_{t}-\beta \lambda u_{x} \xi_{x}+\eta_{t}+\beta \lambda \eta_{x} \\
= & 0 .
\end{aligned}
$$

As before, splitting (29) on derivatives of $u$ and simplifying lead to

$$
\begin{gathered}
\tau_{u}=0, \\
\xi_{u}=0, \\
\eta_{u u}=0, \\
\tau_{x}=0, \\
2 \alpha \eta_{x u} x^{3}-\alpha \xi_{x x} x^{3}-\lambda \xi_{x} x^{2}+\lambda \xi x \\
-\xi_{t} x+\beta \lambda \xi_{x} x-2 \beta \lambda \xi=0, \\
2 x \xi_{x}-2 \xi-x \tau_{t}=0,
\end{gathered}
$$




$$
\begin{aligned}
& x^{q}\left(u \gamma \xi(q-2)+x\left(\gamma \eta-u \gamma \eta_{u}+2 u \gamma \xi_{x}\right)\right) \\
& +x^{2} \alpha \eta_{x x} x+x \lambda \eta_{x}(\beta-x)+x \eta_{t}=0 .
\end{aligned}
$$

Equations (30) and (33) imply that

$$
\tau=\tau(t)
$$

whereas (31) means that $\xi$ does not depend on $u$; that is,

$$
\xi=\xi(t, x)
$$

and (32) gives

$$
\eta(t, x, u)=A(t, x) u+B(t, x),
$$

after integrating twice by $u$ and for some arbitrary functions $A$ and $B$. Substituting (37) and (38) into (35), we get a linear firstorder ODE in $\xi$ which can be easily integrated with respect to $x$ to give

$$
\xi(t, x)=x e(t)+\frac{1}{2} x \tau^{\prime}(t) \ln x,
$$

where $e(t)$ is an arbitrary function of $t$. If we substitute (37), (40), and (39) into (34) and solve the resulting differential equation for $A$, we get

$$
\begin{aligned}
A(t, x)= & \frac{1}{8 \alpha x}\left[\left\{x\left[4 e^{\prime}(t)+\ln x \tau^{\prime \prime}(t)\right]\right.\right. \\
& \left.\left.+2 \tau^{\prime}(t)[x(\alpha+\lambda)-\beta \lambda]\right\} \ln x-4 \beta \lambda e(t)\right] \\
& +f(t),
\end{aligned}
$$

where $f(t)$ is an arbitrary function of $t$. Substituting (41) and (40) into (36), we obtain

$$
\begin{aligned}
8 \alpha^{2} x^{4} B_{x x} & -8 \alpha \lambda x^{3} B_{x}+8 \alpha \beta \lambda x^{2} B_{x}+8 \alpha x^{2} B_{t}+8 \alpha \gamma x^{q+2} B \\
+u( & -4 \alpha x^{2} e^{\prime}(t)-4 \lambda x^{2} e^{\prime}(t)+4 e(t) \\
& \times\left(2 \alpha \gamma q x^{q+2}+\beta \lambda(\beta \lambda-x(2 \alpha+\lambda))\right) \\
& +4 x^{2} e^{\prime \prime}(t) \ln x+8 \alpha x^{2} f^{\prime}(t)+8 \alpha \gamma x^{q+2} \tau^{\prime}(t) \\
& +4 \alpha \gamma q x^{q+2} \tau^{\prime}(t) \ln x-2 \beta^{2} \lambda^{2} \tau^{\prime}(t)-2 \alpha^{2} x^{2} \tau^{\prime}(t) \\
& -4 \alpha \lambda x^{2} \tau^{\prime}(t)+2 \alpha x^{2} \tau^{\prime \prime}(t)-2 \lambda^{2} x^{2} \tau^{\prime}(t) \\
& +x^{2} \tau^{(3)}(t) \ln { }^{2} x+4 \beta \lambda^{2} x \tau^{\prime}(t)+8 \alpha \beta \lambda x \tau^{\prime}(t) \\
& -4 \alpha \beta \lambda x \tau^{\prime}(t) \ln x \\
& \left.+2 \beta^{2} \lambda^{2} \tau^{\prime}(t) \ln x-2 \beta \lambda^{2} x \tau^{\prime}(t) \ln x\right) \\
=0 . \quad &
\end{aligned}
$$

Splitting (42) on $u$ yields

$$
1: B_{t}+\alpha x^{2} B_{x x}+\lambda(\beta-x) B_{x}+\gamma x^{q} B=0,
$$

$u:\left(4 x^{2} e^{\prime \prime}(t)+4 \alpha \gamma q x^{q+2} \tau^{\prime}(t)+2 \beta^{2} \lambda^{2} \tau^{\prime}(t)-4 \alpha \beta \lambda x \tau^{\prime}(t)\right.$

$\left.-2 \beta \lambda^{2} x \tau^{\prime}(t)\right) \ln x-4 \alpha x^{2} e^{\prime}(t)-4 \lambda x^{2} e^{\prime}(t)+4 e(t)$

$$
\begin{aligned}
& \times\left(2 \alpha \gamma q x^{q+2}+\beta \lambda(\beta \lambda-x(2 \alpha+\lambda))\right)+8 \alpha x^{2} f^{\prime}(t) \\
& +8 \alpha \gamma x^{q+2} \tau^{\prime}(t)-2 \beta^{2} \lambda^{2} \tau^{\prime}(t)-2 \alpha^{2} x^{2} \tau^{\prime}(t) \\
& -4 \alpha \lambda x^{2} \tau^{\prime}(t)+2 \alpha x^{2} \tau^{\prime \prime}(t)-2 \lambda^{2} x^{2} \tau^{\prime}(t) \\
& +x^{2} \tau^{\prime \prime \prime}(t) \ln ^{2} x+8 \alpha \beta \lambda x \tau^{\prime}(t)+4 \beta \lambda^{2} x \tau^{\prime}(t)
\end{aligned}
$$$$
=0 \text {. }
$$

Rewriting (44) we get our classifying equation as

$$
\begin{aligned}
b_{0}(t) & +b_{1}(t) \ln x+x\left(b_{2}(t)+b_{3}(t) \ln x\right) \\
& +x^{2}\left(b_{4}(t)+b_{5}(t) \ln x+b_{6}(t) \ln ^{2} x\right) \\
& +x^{q+2}\left(b_{7}(t)+b_{8}(t) \ln x\right)=0,
\end{aligned}
$$

where

$$
\begin{gathered}
b_{0}(t)=4 \beta^{2} \lambda^{2} e(t)-2 \beta^{2} \lambda^{2} \tau^{\prime}(t), \\
b(1)=2 \beta^{2} \lambda^{2} \tau^{\prime}(t), \\
b_{2}(t)=-4 \beta \lambda(2 \alpha+\lambda)\left(e(t)-\tau^{\prime}(t)\right), \\
b_{3}(t)=-2 \beta \lambda(2 \alpha+\lambda) \tau^{\prime}(t), \\
b_{4}(t)=8 \alpha f^{\prime}(t)-4(\alpha+\lambda) e^{\prime}(t) \\
-2(\alpha+\lambda)^{2} \tau^{\prime}(t)+2 \alpha \tau^{\prime \prime}(t), \\
b_{5}(t)=4 e^{\prime \prime}(t), \\
b_{6}(t)=\tau^{\prime \prime \prime}(t), \\
b_{7}(t)=4 \alpha \gamma\left(2 q e(t)+2 \tau^{\prime}(t)\right), \\
b_{8}(t)=4 \alpha \gamma q \tau^{\prime}(t) .
\end{gathered}
$$

\section{Results of Group Classification}

We note that our classifying equations (27) and (45) are satisfied if we choose

$$
c(t)=e(t)=0, \quad d(t)=f(t)=c_{2}, \quad \tau(t)=c_{1},
$$

for some constants $c_{1}$ and $c_{2}$. Thus using these values, for both cases, the coefficients of the infinitesimal operator are

$$
\tau=c_{1}, \quad \xi=0, \quad \eta=c_{2} u+B(t, x),
$$

where $B(t, x)$ is any solution of (4). 
Case $0(\alpha, \gamma, \lambda, \beta, p, q$ arbitrary). We obtain the following Lie point symmetries:

$$
X_{1}=\frac{\partial}{\partial t}, \quad X_{2}=u \frac{\partial}{\partial u}, \quad X_{B}=B(t, x) \frac{\partial}{\partial u},
$$

where the symmetry associated with $B$ is the solution symmetry. Lie symmetries (49) generate what is called the principal Lie algebra.

By equating the powers of $x$ in (27) and solving for $p$ we infer that possible extensions of the principal Lie algebra are possible for the following values of $p$ :

$$
0,1, \frac{3}{2}, \frac{4}{3}, \frac{6}{5}, \frac{8}{5}, \frac{8}{3}, 3,4,6
$$

In this paper, we consider $p=0,1$, and 2 , as these values of $p$ provide us with very important equations in mathematics of finance. For example, when $p=2$ and $q=0$, we have the Black-Scholes equation. We obtain the Vasicek equation when $p=0$ and $q=1$ and CIR equation when $p=1$ and $q=1$.

We show only the different combinations of parameters which extend the principal Lie algebra.

Case $1(p=0)$

Case $1.1(q=0)$. In this case, the principal Lie algebra extends by the following Lie point symmetries:

$$
\begin{gathered}
X_{3}=\frac{e^{2 \lambda t}}{2 \lambda} \frac{\partial}{\partial t}+e^{2 \lambda t}\left(\frac{x}{2}-\frac{\beta}{2}\right) \frac{\partial}{\partial x} \\
+u e^{2 \lambda t}\left(\frac{\beta^{2} \lambda}{2 \alpha}-\frac{\gamma}{2 \lambda}+\frac{\lambda x^{2}}{2 \alpha}-\frac{\beta \lambda x}{\alpha}-\frac{1}{2}\right) \frac{\partial}{\partial u}, \\
X_{4}=\frac{e^{-2 \lambda t}}{2 \lambda}\left[-\frac{\partial}{\partial t}+\lambda(x-\beta) \frac{\partial}{\partial x}+\gamma u \frac{\partial}{\partial u}\right], \\
X_{5}=e^{\lambda t}\left[\frac{\partial}{\partial x}+\frac{\lambda}{\alpha} u(x-\beta) \frac{\partial}{\partial u}\right] \\
X_{6}=e^{-\lambda t} \frac{\partial}{\partial x} .
\end{gathered}
$$

Case $1.2(q=1)$. The principal Lie algebra extends by

$$
\begin{aligned}
& X_{3}= \frac{e^{2 \lambda t}}{2 \lambda} \frac{\partial}{\partial t}+e^{2 \lambda t}\left(-\frac{\alpha \gamma}{\lambda^{2}}-\frac{\beta}{2}+\frac{x}{2}\right) \frac{\partial}{\partial x} \\
&+u e^{2 \lambda t}\left(\frac{\beta^{2} \lambda}{2 \alpha}+\frac{\alpha \gamma^{2}}{2 \lambda^{3}}+\frac{\beta \gamma}{\lambda}+\frac{\lambda x^{2}}{2 \alpha}-\frac{\beta \lambda x}{\alpha}\right. \\
&\left.-\frac{3 \gamma x}{2 \lambda}-\frac{1}{2}\right) \frac{\partial}{\partial u},
\end{aligned}
$$

$$
\begin{gathered}
X_{4}=\frac{e^{-2 \lambda t}}{2 \lambda}\left[-\frac{\partial}{\partial t}+\frac{1}{\lambda}\left(\lambda^{2}(x-\beta)-2 \alpha \gamma\right) \frac{\partial}{\partial x}\right. \\
\left.+\frac{\gamma u}{\lambda^{2}}\left(\lambda^{2} x-\alpha \gamma\right) \frac{\partial}{\partial u}\right], \\
X_{5}=e^{\lambda t}\left[\frac{\partial}{\partial x}+\frac{u}{\alpha \lambda}\left(-\alpha \gamma-\beta \lambda^{2}+\lambda^{2} x\right) \frac{\partial}{\partial u}\right], \\
X_{6}=e^{-\lambda t}\left[\frac{\partial}{\partial x}+\frac{\gamma u}{\lambda} \frac{\partial}{\partial u}\right] .
\end{gathered}
$$

It should be noted that this case results in the Vasicek equation [26].

Case $1.3(q=2)$. (1) Consider $\alpha \neq \lambda^{2} / 4 \gamma$. The additional Lie point symmetries are given by

$$
\begin{aligned}
X_{3}= & \frac{e^{2 \kappa t}}{2 \kappa} \frac{\partial}{\partial t}+e^{2 \kappa t}\left(\frac{1}{2} x-\frac{\beta \lambda^{2}}{2 \kappa}\right) \frac{\partial}{\partial x} \\
+ & \frac{u e^{2 \kappa t}}{4 \alpha \kappa^{3}}\left(-2 \alpha \beta^{2} \gamma \lambda^{2}+(\kappa+\lambda)\right. \\
& \left.\times\left((\kappa x-\beta \lambda)\left(\kappa^{2} x-\beta \lambda^{2}\right)-\alpha \kappa^{2}\right)\right) \frac{\partial}{\partial u}, \\
X_{4}= & -\frac{e^{-2 \kappa t}}{2 \kappa} \frac{\partial}{\partial t}+\frac{e^{-2 \kappa t}}{2 \kappa^{2}}\left(\kappa^{2} x-\beta \lambda^{2}\right) \frac{\partial}{\partial x} \\
& -\frac{u e^{-2 t \kappa}}{4 \alpha \kappa^{3}}\left(\alpha \lambda\left(2 \beta^{2} \gamma \lambda+\kappa(\kappa+\lambda)\right)-4 \alpha^{2} \gamma \kappa\right. \\
X_{5}= & e^{\kappa t}\left[\frac{\partial}{\partial x}+\frac{1}{2 \alpha \kappa} u(\kappa+\lambda)(x \kappa+\beta \lambda) \frac{\partial}{\partial u}\right], \\
X_{6}= & e^{-\kappa t}\left[\frac{\partial}{\partial x}-\frac{1}{2 \alpha \kappa} u(\kappa-\lambda)(x \kappa-\beta \lambda) \frac{\partial}{\partial u}\right],
\end{aligned}
$$

where $\kappa=\sqrt{\lambda^{2}-4 \alpha \gamma}$.

(2) Consider $\alpha=\lambda^{2} / 4 \gamma$. The additional Lie point symmetries are

$$
\begin{aligned}
X_{3}= & t \frac{\partial}{\partial t}+\left(\frac{x}{2}-\frac{3}{4} \beta \lambda^{2} t^{2}\right) \frac{\partial}{\partial x} \\
+ & u\left(\frac{1}{2} \beta^{2} \gamma \lambda^{2} t^{3}+\frac{3}{2} \beta^{2} \gamma \lambda t^{2}-\frac{3}{2} \beta \gamma \lambda t^{2} x\right. \\
& \left.+\beta^{2} \gamma t-\frac{\lambda t}{2}-3 \beta \gamma t x+\frac{\gamma x^{2}}{\lambda}-\frac{\beta \gamma x}{\lambda}\right) \frac{\partial}{\partial u},
\end{aligned}
$$




$$
\begin{aligned}
& X_{4}= t^{2} \frac{\partial}{\partial t}+\left(t x-\frac{1}{2} \beta \lambda^{2} t^{3}\right) \frac{\partial}{\partial x} \\
&+ u\left(\frac{1}{4} \beta^{2} \gamma \lambda^{2} t^{4}+\beta^{2} \gamma \lambda t^{3}-\beta \gamma \lambda t^{3} x+\beta^{2} \gamma t^{2}-\frac{\lambda t^{2}}{2}\right. \\
&\left.-3 \beta \gamma t^{2} x+\frac{2 \gamma t x^{2}}{\lambda}-\frac{2 \beta \gamma t x}{\lambda}-\frac{t}{2}+\frac{\gamma x^{2}}{\lambda^{2}}\right) \frac{\partial}{\partial u}, \\
& X_{5}=\frac{\partial}{\partial x}+u\left(\frac{2 \gamma x}{\lambda}-2 \beta \gamma t\right) \frac{\partial}{\partial u} \\
& X_{6}=t \frac{\partial}{\partial x}+u\left(-\beta \gamma t^{2}-\frac{2 \beta \gamma t}{\lambda}+\frac{2 \gamma t x}{\lambda}+\frac{2 \gamma x}{\lambda^{2}}\right) \frac{\partial}{\partial u} .
\end{aligned}
$$

Case $2(p=1)$

Case $2.1(q=0)$. (1) Consider $\beta \neq \alpha / 2 \lambda$ and $\beta \neq 3 \alpha / 2 \lambda$. The principal Lie algebra is extended by

$$
\begin{gathered}
X_{3}=e^{\lambda t}\left[\frac{1}{\lambda} \frac{\partial}{\partial t}+x \frac{\partial}{\partial x}+u\left(\frac{\lambda x}{\alpha}-\frac{\beta \lambda}{\alpha}-\frac{\gamma}{\lambda}\right) \frac{\partial}{\partial u}\right], \\
X_{4}=\frac{e^{-\lambda t}}{\lambda}\left[-\frac{\partial}{\partial t}+x \lambda \frac{\partial}{\partial x}+\gamma u \frac{\partial}{\partial u}\right] .
\end{gathered}
$$

(2) Consider $\beta=\alpha / 2 \lambda$. The additional Lie point symmetries are

$$
\begin{gathered}
X_{3}=e^{\lambda t}\left[\frac{1}{\lambda} \frac{\partial}{\partial t}+x \frac{\partial}{\partial x}+u\left(\frac{\lambda x}{\alpha}-\frac{\gamma}{\lambda}-\frac{1}{2}\right) \frac{\partial}{\partial u}\right], \\
X_{4}=\frac{e^{-\lambda t}}{\lambda}\left[-\frac{\partial}{\partial t}+x \lambda \frac{\partial}{\partial x}+\gamma u \frac{\partial}{\partial u}\right], \\
X_{5}=\sqrt{x} e^{\lambda t / 2} \frac{\partial}{\partial x}+\frac{\lambda u \sqrt{x} e^{\lambda t / 2}}{\alpha} \frac{\partial}{\partial u}, \\
X_{6}=\sqrt{x} e^{-\lambda t / 2} \frac{\partial}{\partial x} .
\end{gathered}
$$

(3) Consider $\beta=3 \alpha / 2 \lambda$. The Lie point symmetries that extend the principal Lie algebra are

$$
\begin{gathered}
X_{3}=\frac{e^{\lambda t}}{\lambda} \frac{\partial}{\partial t}+x e^{\lambda t} \frac{\partial}{\partial x}+e^{\lambda t}\left(\frac{\lambda x}{\alpha}-\frac{\gamma}{\lambda}-\frac{3}{2}\right) u \frac{\partial}{\partial u}, \\
X_{4}=-\frac{e^{-\lambda t}}{\lambda} \frac{\partial}{\partial t}+x e^{-\lambda t} \frac{\partial}{\partial x}+\frac{\gamma u e^{-\lambda t}}{\lambda} \frac{\partial}{\partial u}, \\
X_{5}=\sqrt{x} e^{\lambda t / 2} \frac{\partial}{\partial x}+e^{\lambda t / 2}\left(\frac{\lambda \sqrt{x}}{\alpha}-\frac{1}{2 \sqrt{x}}\right) u \frac{\partial}{\partial u}, \\
X_{6}=\sqrt{x} e^{-\lambda t / 2} \frac{\partial}{\partial x}-\frac{u e^{-\lambda t / 2}}{2 \sqrt{x}} \frac{\partial}{\partial u} .
\end{gathered}
$$

Case $2.2(q=1 / 2)$. This has two subcases.

(1) Consider $\beta=\alpha / 2 \lambda$. This case results in the following extra Lie point symmetries:

$$
\begin{aligned}
X_{3}= & \frac{e^{\lambda t}}{\lambda} \frac{\partial}{\partial t}+e^{\lambda t}\left(x-\frac{2 \alpha \gamma \sqrt{x}}{\lambda^{2}}\right) \frac{\partial}{\partial x} \\
& +u e^{\lambda t}\left(\frac{\lambda x}{\alpha}-\frac{3 \gamma \sqrt{x}}{\lambda}+\frac{\alpha \gamma^{2}}{\lambda^{3}}-\frac{1}{2}\right) \frac{\partial}{\partial u} \\
X_{4}= & -\frac{e^{-\lambda t}}{\lambda} \frac{\partial}{\partial t}+e^{-\lambda t}\left(x-\frac{2 \alpha \gamma \sqrt{x}}{\lambda^{2}}\right) \frac{\partial}{\partial x} \\
& +u e^{-\lambda t}\left(\frac{\gamma \sqrt{x}}{\lambda}-\frac{\alpha \gamma^{2}}{\lambda^{3}}\right) \frac{\partial}{\partial u} \\
X_{5}= & \sqrt{x} e^{\lambda t / 2} \frac{\partial}{\partial x}+u e^{\lambda t / 2}\left(\frac{\lambda \sqrt{x}}{\alpha}-\frac{\gamma}{\lambda}\right) \frac{\partial}{\partial u} \\
X_{6} & =\sqrt{x} e^{-\lambda t / 2} \frac{\partial}{\partial x}+\frac{\gamma u e^{-\lambda t / 2}}{\lambda} \frac{\partial}{\partial u} .
\end{aligned}
$$

(2) Consider $\beta=3 \alpha / 2 \lambda$. The extra Lie point symmetries are

$$
\begin{gathered}
X_{3}=\frac{e^{\lambda t}}{\lambda} \frac{\partial}{\partial t}+e^{\lambda t}\left(x-\frac{2 \alpha \gamma \sqrt{x}}{\lambda^{2}}\right) \frac{\partial}{\partial x} \\
+e^{\lambda t}\left(\frac{\alpha \gamma^{2}}{\lambda^{3}}-\frac{3}{2}+\frac{\alpha \gamma}{\lambda^{2} \sqrt{x}}+\frac{\lambda x}{\alpha}-\frac{3 \gamma \sqrt{x}}{\lambda}\right) u \frac{\partial}{\partial u}, \\
X_{4}=-\frac{e^{-\lambda t}}{\lambda} \frac{\partial}{\partial t}+e^{-\lambda t}\left(x-\frac{2 \alpha \gamma \sqrt{x}}{\lambda^{2}}\right) \frac{\partial}{\partial x} \\
+e^{-\lambda t}\left(-\frac{\alpha \gamma^{2}}{\lambda^{3}}+\frac{\alpha \gamma}{\lambda^{2} \sqrt{x}}+\frac{\gamma \sqrt{x}}{\lambda}\right) u \frac{\partial}{\partial u}, \\
X_{5}=\sqrt{x} e^{-\frac{\lambda t}{2}} \frac{\partial}{\partial x}+e^{-\frac{\lambda t}{2}}\left(\frac{\gamma}{\lambda}-\frac{1}{2 \sqrt{x}}\right) u \frac{\partial}{\partial u}, \\
X_{6}=\sqrt{x} e \frac{\lambda t}{2} \frac{\partial}{\partial x}+e^{\frac{\lambda t}{2}}\left(\frac{\lambda \sqrt{x}}{\alpha}-\frac{\gamma}{\lambda}-\frac{1}{2 \sqrt{x}}\right) u \frac{\partial}{\partial u} .
\end{gathered}
$$

Case $2.3(q=1)$. This leads to six subcases.

(1) Consider $\beta \neq \alpha / 2 \lambda$ and $\beta \neq 3 \alpha / 2 \lambda$ and $\alpha \neq \lambda^{2} / 4 \gamma$. The principal Lie algebra is extended by

$$
\begin{aligned}
X_{3}=\frac{e^{t \kappa}}{\kappa} \frac{\partial}{\partial t}+ & x e^{t \kappa} \frac{\partial}{\partial x}+\frac{u e^{t \kappa}}{2 \alpha}\left(x \kappa-\frac{\beta \lambda^{2}}{\kappa}+\lambda x-\beta \lambda\right) \frac{\partial}{\partial u}, \\
X_{4}= & -\frac{e^{-t \kappa}}{\kappa} \frac{\partial}{\partial t}+x e^{-t \kappa} \frac{\partial}{\partial x} \\
& +\frac{u e^{-t \kappa}}{2 \alpha}\left(\frac{\beta \lambda^{2}}{\kappa}-\beta \lambda+\lambda x-x \kappa\right) \frac{\partial}{\partial u},
\end{aligned}
$$


where $\kappa=\sqrt{\lambda^{2}-4 \alpha \gamma}$. We note that this case gives us the CIR equation $[26,27]$.

(2) Consider $\alpha=\lambda^{2} / 4 \gamma, \beta \neq \alpha / 2 \lambda$, and $\beta \neq 3 \alpha / 2 \lambda$. The extra symmetries that extend the principal Lie algebra are given by

$$
\begin{gathered}
X_{3}=t \frac{\partial}{\partial t}+x \frac{\partial}{\partial x}+\gamma u\left(\frac{2 x}{\lambda}-2 \beta t\right) \frac{\partial}{\partial u} \\
X_{4}=t^{2} \frac{\partial}{\partial t}+2 t x \frac{\partial}{\partial x}+\gamma u\left(\frac{4 t x}{\lambda}+\frac{4 x}{\lambda^{2}}-2 \beta t^{2}-\frac{4 \beta t}{\lambda}\right) \frac{\partial}{\partial u} .
\end{gathered}
$$

(3) Consider $\alpha=\lambda^{2} / 4 \gamma, \beta=\alpha / 2 \lambda$. Additional Lie point symmetries are

$$
\begin{gathered}
X_{3}=t \frac{\partial}{\partial t}+x \frac{\partial}{\partial x}+u\left(\frac{2 \gamma x}{\lambda}-\frac{1}{4} \lambda t\right) \frac{\partial}{\partial u} \\
X_{4}=t^{2} \frac{\partial}{\partial t}+2 t x \frac{\partial}{\partial x}+u\left(\frac{4 \gamma t x}{\lambda}-\frac{1}{4} \lambda t^{2}-\frac{1}{2} t+\frac{4 \gamma x}{\lambda^{2}}\right) \frac{\partial}{\partial u} \\
X_{5}=\sqrt{x} \frac{\partial}{\partial x}+\frac{2 \gamma u \sqrt{x}}{\lambda} \frac{\partial}{\partial u} \\
X_{6}=t \sqrt{x} \frac{\partial}{\partial x}+2 \gamma u \sqrt{x}\left(\frac{2}{\lambda^{2}}+\frac{t}{\lambda}\right) \frac{\partial}{\partial u}
\end{gathered}
$$

(4) Consider $\alpha=\lambda^{2} / 4 \gamma, \beta=3 \alpha / 2 \lambda$. Additional Lie point symmetries to the principal Lie algebra are given by

$$
\begin{gathered}
X_{3}=t \frac{\partial}{\partial t}+x \frac{\partial}{\partial x}+u\left(\frac{2 \gamma x}{\lambda}-\frac{3}{4} \lambda t\right) \frac{\partial}{\partial u} \\
X_{4}=t^{2} \frac{\partial}{\partial t}+2 t x \frac{\partial}{\partial x}+u\left(\frac{4 \gamma t x}{\lambda}-\frac{3}{2} t+\frac{4 \gamma x}{\lambda^{2}}-\frac{3}{4} \lambda t^{2}\right) \frac{\partial}{\partial u}, \\
X_{5}=\sqrt{x} \frac{\partial}{\partial x}+u\left(\frac{2 \gamma \sqrt{x}}{\lambda}-\frac{1}{2 \sqrt{x}}\right) \frac{\partial}{\partial u} \\
X_{6}=t \sqrt{x} \frac{\partial}{\partial x}+u\left(\frac{2 \gamma t \sqrt{x}}{\lambda}-\frac{t}{2 \sqrt{x}}+\frac{4 \gamma \sqrt{x}}{\lambda^{2}}\right) \frac{\partial}{\partial u} .
\end{gathered}
$$

(5) Consider $\beta=\alpha / 2 \lambda, \alpha \neq \lambda^{2} / 4 \gamma$. The extra Lie point symmetries are

$$
\begin{gathered}
X_{3}=\frac{e^{t \kappa}}{\kappa} \frac{\partial}{\partial t}+x e^{t \kappa} \frac{\partial}{\partial x}+u e^{t \kappa}\left(\frac{x \kappa}{2 \alpha}-\frac{1}{4}-\frac{\lambda}{4 \kappa}+\frac{\lambda x}{2 \alpha}\right) \frac{\partial}{\partial u}, \\
X_{4}=-\frac{e^{-t \kappa}}{\kappa} \frac{\partial}{\partial t}+x e^{-t \kappa} \frac{\partial}{\partial x} \\
+u e^{-t \kappa}\left(\frac{\lambda}{4 \kappa}-\frac{1}{4}+\frac{\lambda x}{2 \alpha}-\frac{x \kappa}{2 \alpha}\right) \frac{\partial}{\partial u} \\
X_{5}=\sqrt{x} e^{(1 / 2) t \kappa} \frac{\partial}{\partial x}+u \sqrt{x} e^{(1 / 2) t \kappa} \frac{\lambda+\kappa}{2 \alpha} \frac{\partial}{\partial u}, \\
X_{6}=\sqrt{x} e^{-(1 / 2) t \kappa} \frac{\partial}{\partial x}+u \sqrt{x} e^{-(1 / 2) t \kappa} \frac{\lambda-\kappa}{2 \alpha} \frac{\partial}{\partial u},
\end{gathered}
$$

(6) Consider $\beta=3 \alpha / 2 \lambda, \alpha \neq \lambda^{2} / 4 \gamma$. The principal Lie algebra is extended by

$$
\begin{gathered}
X_{3}=\frac{e^{\kappa t}}{\kappa} \frac{\partial}{\partial t}+x e^{\kappa t} \frac{\partial}{\partial x}+e^{\kappa t}\left(\frac{\kappa x}{2 \alpha}-\frac{3 \lambda}{4 \kappa}-\frac{3}{4}+\frac{\lambda x}{2 \alpha}\right) u \frac{\partial}{\partial u}, \\
X_{4}=-\frac{e^{-\kappa t}}{\kappa} \frac{\partial}{\partial t}+x e^{-\kappa t} \frac{\partial}{\partial x} \\
+e^{-\kappa t}\left(\frac{3 \lambda}{4 \kappa}-\frac{3}{4}+\frac{\lambda x}{2 \alpha}-\frac{\kappa x}{2 \alpha}\right) u \frac{\partial}{\partial u}, \\
X_{5}=\sqrt{x} e^{\kappa t / 2} \frac{\partial}{\partial x}+e^{\kappa t / 2}\left(\frac{\lambda \sqrt{x}}{2 \alpha}+\frac{\kappa \sqrt{x}}{2 \alpha}-\frac{1}{2 \sqrt{x}}\right) u \frac{\partial}{\partial u}, \\
X_{6}=\sqrt{x} e^{-\kappa t / 2} \frac{\partial}{\partial x}+e^{-\kappa t / 2}\left(\frac{\lambda \sqrt{x}}{2 \alpha}-\frac{\kappa \sqrt{x}}{2 \alpha}-\frac{1}{2 \sqrt{x}}\right) u \frac{\partial}{\partial u},
\end{gathered}
$$

where $\kappa=\sqrt{\lambda^{2}-4 \alpha \gamma}$.

Case $3(p=2)$. We can conclude from (45) that $q$ can only take the value 0 . Proceeding as before, we find that the principal Lie algebra extends for the case when $\beta=0$ by the following symmetry operators:

$$
\begin{array}{rl}
X_{3}= & t \frac{\partial}{\partial t}+\frac{1}{2} x \ln x \frac{\partial}{\partial x} \\
+ & u\left(\frac{\lambda^{2} t}{4 \alpha}+\frac{1}{4} \alpha t-\gamma t+\frac{1}{2} \lambda t+\frac{\lambda \ln x}{4 \alpha}+\frac{1}{4} \ln x\right) \frac{\partial}{\partial u} \\
X_{4}= & t^{2} \frac{\partial}{\partial t}+t x \ln x \frac{\partial}{\partial x} \\
& +u\left(\frac{\lambda^{2} t^{2}}{4 \alpha}+\frac{1}{4} \alpha t^{2}-\gamma t^{2}+\frac{1}{2} \lambda t^{2}-\frac{1}{2} t\right. \\
& \left.+\frac{\lambda t \ln x}{2 \alpha}+\frac{1}{2} t \ln x+\frac{\ln 2 x}{4 \alpha}\right) \frac{\partial}{\partial u} \\
X_{5}=x \frac{\partial}{\partial x} & \\
X_{6} & t x \frac{\partial}{\partial x}+u\left(\frac{\lambda t}{2 \alpha}+\frac{1}{2} t+\frac{\ln x}{2 \alpha}\right) \frac{\partial}{\partial u} .
\end{array}
$$

This case gives us the Black-Scholes equation [30].

\section{Symmetry Reductions and Group Invariant Solutions}

We obtain symmetry reductions [31] and construct group invariant solutions of Case 2.1(1), that is, when $p=1, q=0$, $\beta \neq \alpha /(2 \lambda)$, and $\beta \neq 3 \alpha /(2 \lambda)$, while all other constants in (4) are arbitrary. Equation (4) is then given by

$$
u_{t}+\alpha x u_{x x}+\lambda(\beta-x) u_{x}+\gamma u=0 .
$$


Case $2.1\left(X_{1}\right)$. The operator $X_{1}$ results in two invariants $J_{1}=$ $x$ and $J_{2}=u$. The group invariant solution is given by $u=$ $f(x)$, where $f$ solves

$$
\alpha x f^{\prime \prime}(x)+\lambda(\beta-x) f^{\prime}(x)+\gamma f(x)=0 .
$$

Case $2.2\left(X_{3}\right)$. The operator $X_{3}$ gives the following two invariants:

$$
J_{1}=x e^{-\lambda t}, \quad J_{2}=u \exp \left\{\frac{\alpha \gamma t+\beta \lambda^{2} t-\lambda x}{\alpha}\right\} .
$$

Hence, the invariant solution of (67) under $X_{3}$ is given by

$$
u=f\left(x e^{-\lambda t}\right) \exp \left\{\frac{\lambda x-t\left(\alpha \gamma+\beta \lambda^{2}\right)}{\alpha}\right\},
$$

where $f$ satisfies

$$
\alpha z f^{\prime \prime}(z)+\beta \lambda f^{\prime}(z)=0, \quad z=x e^{-\lambda t} .
$$

The solution of the above equation is

$$
f(z)=\frac{c_{1} \alpha z^{(\alpha-\beta \lambda) / \alpha}}{\alpha-\beta \lambda}+c_{2}
$$

where $c_{1}$ and $c_{2}$ are arbitrary constants. Hence, the group invariant solution under $X_{3}$ is

$$
\begin{aligned}
u(t, x)= & {\left[\frac{c_{1} \alpha\left(x e^{-\lambda t}\right)^{(\alpha-\beta \lambda) / \alpha}}{\alpha-\beta \lambda}+c_{2}\right] } \\
& \times \exp \left\{\frac{\lambda x-t\left(\alpha \gamma+\beta \lambda^{2}\right)}{\alpha}\right\} .
\end{aligned}
$$

Case $2.3\left(X_{4}\right)$. The symmetry operator $X_{4}$ gives the invariants $J_{1}=x e^{\lambda t}$ and $J_{2}=u e^{\gamma t}$. Thus, the group invariant solution in this case is given by

$$
u(t, x)=\left[\frac{c_{1} \alpha\left(x e^{\lambda t}\right)^{(\alpha-\beta \lambda) / \alpha}}{\alpha-\beta \lambda}+c_{2}\right] e^{-\gamma t},
$$

where $c_{1}$ and $c_{2}$ are arbitrary constants.

It should be noted that the operators $X_{2}$ and $X_{B}$ do not provide invariant solutions.

\section{Conclusions}

In this paper we carried out group classification of the general bond-option pricing $\operatorname{PDE}(4)$ for $p=0,1$, and 2 . The principal Lie algebra was found to be three-dimensional. These values of $p$ resulted in 16 cases, which extended the principal Lie algebra. We presented the Lie point symmetries for each case. Three cases gave us the option pricing equations, which were given by Cases 1.1, 2.1(1), and 3. In the last case, Black-Scholes equation was recovered. Seven bond pricing equations were obtained and these were Case 1.2 and Cases 2.3(1)-2.3(6). Cases 1.2 and 2.3(1) were found to be the Vasicek and CIR equations, respectively. Finally, symmetry reductions and construction of group invariant solutions for Case 2.1(1) were presented.

\section{Conflict of Interests}

The authors declare that there is no conflict of interests regarding the publication of this paper.

\section{References}

[1] L. Bachelier, "Théorie de la spéculation," Annales Scientifiques de l'École Normale Supérieure, vol. 3, pp. 21-86, 1900.

[2] F. Black and M. Scholes, "The pricing of options and corporate liabilities," Journal of Political Economy, vol. 81, pp. 637-654, 1973.

[3] R. C. Merton, "Theory of rational option pricing," Bell Journal of Economics and Management Science, vol. 4, no. 1, pp. 141-183, 1973.

[4] O. Vasicek, "An equilibrium characterization of the term structure," Journal of Financial Economics, vol. 5, no. 2, pp. 177-188, 1977.

[5] L. U. Dothan, "On the term structure of interest rates," Journal of Financial Economics, vol. 6, no. 1, pp. 59-69, 1978.

[6] J. C. Cox, J. E. Ingersoll, and S. A. Ross, "A theory of the term structure of interest rates," Econometrica, vol. 53, pp. 385-407, 1985.

[7] F. A. Longstaff, "A nonlinear general equilibrium model of the term structure of interest rates," Journal of Financial Economics, vol. 23, no. 2, pp. 195-224, 1989.

[8] T. S. Y. Ho and S. Lee, "Term structure movements and pricing interest rate contingent claims," The Journal of Finance, pp. 10111029, 1986, 41.

[9] F. Black, E. Derman, and W. Toy, "A one-factor model of interest rates and its applications to treasury bond options," Financial Analysts Journal, vol. 46, pp. 33-39, 1990.

[10] J. Hull and A. White, "Pricing interest rate derivatives securities," Review of Financial Studies, vol. 3, pp. 573-592, 1990.

[11] F. Black and P. Karasinski, "Bond and option pricing when short rates are lognormal," Financial Analysts Journal, vol. 47, pp. 5259, 1991.

[12] D. Heath, R. Jarrow, and A. Morton, "Bond pricing and the term structure of interest rates: a new methodology for contingent claims valuation," Econometrica, vol. 60, pp. 77-105, 1992.

[13] N. H. Ibragimov, Ed., CRC Handbook of Lie Group Analysis of Differential Equations, vol. 1-3, CRC Press, Boca Raton, Fla, USA, 1994-1996.

[14] M. Molati and C. M. Khalique, "Group classification and exact solutions of a generalized Emden-Fowler equation," International Journal of Non-Linear Mechanics, vol. 27, pp. 785-791, 2012.

[15] C. M. Khalique and B. Muatjetjeja, "Lie group classification of the generalized Lane-Emden equation," Applied Mathematics and Computation, vol. 210, no. 2, pp. 405-410, 2009.

[16] M. Molati and F. M. Mahomed, "Symmetry classification of collapsible tube model incorporating tension," Communications in Nonlinear Science and Numerical Simulation, vol. 17, no. 1, pp. 93-99, 2012.

[17] N. M. Ivanova, C. Sophocleous, and P. G. L. Leach, "Group classification of a class of equations arising in financial mathematics," Journal of Mathematical Analysis and Applications, vol. 372, no. 1, pp. 273-286, 2010.

[18] M. Molati and C. M. Khalique, "Lie symmetry analysis of the time-variable coefficient B-BBM equation," Advances in Differential Equations, vol. 2012, no. 212, 2012. 
[19] M. Molati and C. M. Khalique, "Symmetry classification and invariant solutions of the variable coefficient BBM equation," Applied Mathematics and Computation, vol. 219, pp. 7917-7922, 2013.

[20] W. Sinkala, P. G. L. Leach, and J. G. O'Hara, "Invariance properties of a general bond-pricing equation," Journal of Differential Equations, vol. 244, no. 11, pp. 2820-2835, 2008.

[21] S. Lie, "Uber die integration durch bestimmte integrale von einer klasse linearer partieller differentialgleichungen," Archiv for Mathematik og Naturvidenskab, vol. 6, pp. 328-368, 1881.

[22] I. K. Johnpillai and F. M. Mahomed, "Singular invariant equation for the $(1+1)$ Fokker-Planck equation," Journal of Physics A: Mathematical and General, vol. 34, no. 49, pp. 11033-11051, 2001.

[23] F. M. Mahomed, "Complete invariant characterization of scalar linear $(1+1)$ parabolic equations," Journal of Nonlinear Mathematical Physics, vol. 15, no. 1, pp. 112-123, 2008.

[24] J. Goard, "New solutions to the bond-pricing equation via Lie's classical method," Mathematical and Computer Modelling, vol. 32, no. 3-4, pp. 299-313, 2000.

[25] C. A. Pooe, F. M. Mahomed, and C. W. Soh, "Fundamental solutions for zero-coupon bond pricing models," Nonlinear Dynamics, vol. 36, no. 1, pp. 69-76, 2004.

[26] W. Sinkala, P. G. L. Leach, and J. G. O’Hara, “Zero-coupon bond prices in the Vasicek and CIR models: their computation as group-invariant solutions," Mathematical Methods in the Applied Sciences, vol. 31, no. 6, pp. 665-678, 2008.

[27] W. Sinkala, P. G. L. Leach, and J. G. O’Hara, "An optimal system and group-invariant solutions of the Cox-Ingersoll-Ross pricing equation," Applied Mathematics and Computation, vol. 201, no. 1-2, pp. 95-107, 2008.

[28] F. M. Mahomed, K. S. Mahomed, R. Naz, and E. Momoniat, "Invariant approaches to equations of finance," Mathematical and Computational Applications, vol. 18, pp. 244-250, 2013.

[29] S. Dimas, K. Andriopoulos, D. Tsoubelis, and P. G. L. Leach, "Complete specification of some partial differential equations that arise in financial mathematics," Journal of Nonlinear Mathematical Physics, vol. 16, no. 1, pp. 73-92, 2009.

[30] R. K. Gazizov and N. H. Ibragimov, "Lie symmetry analysis of differential equations in finance," Nonlinear Dynamics, vol. 17, no. 4, pp. 387-407, 1998.

[31] H. Liu and J. Li, "Symmetry reductions, dynamical behavior and exact explicit solutions to the Gordon types of equations," Journal of Computational and Applied Mathematics, vol. 257, pp. 144-156, 2014. 


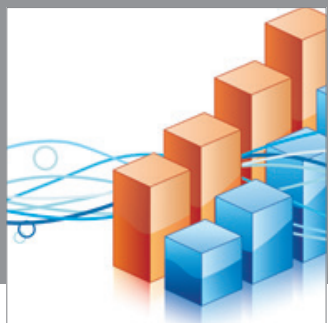

Advances in

Operations Research

mansans

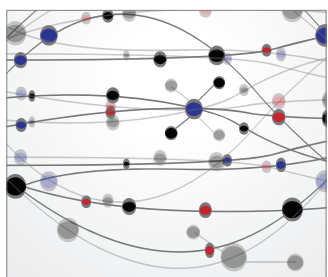

The Scientific World Journal
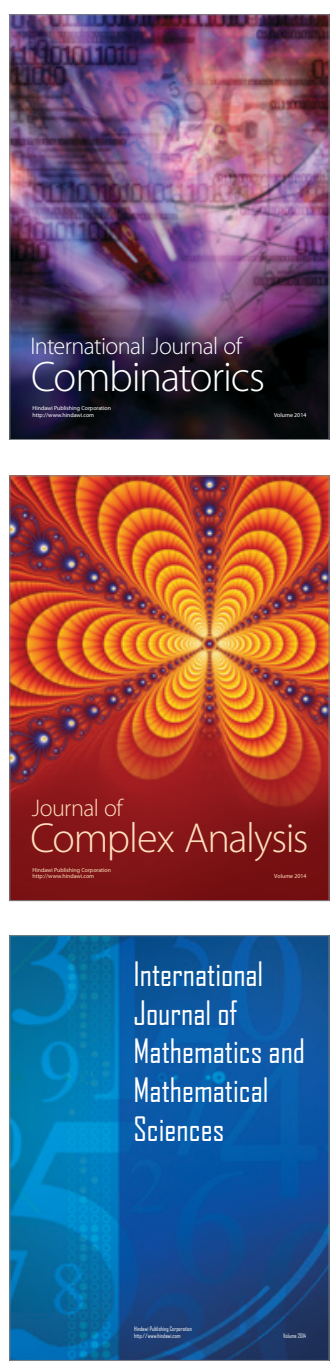
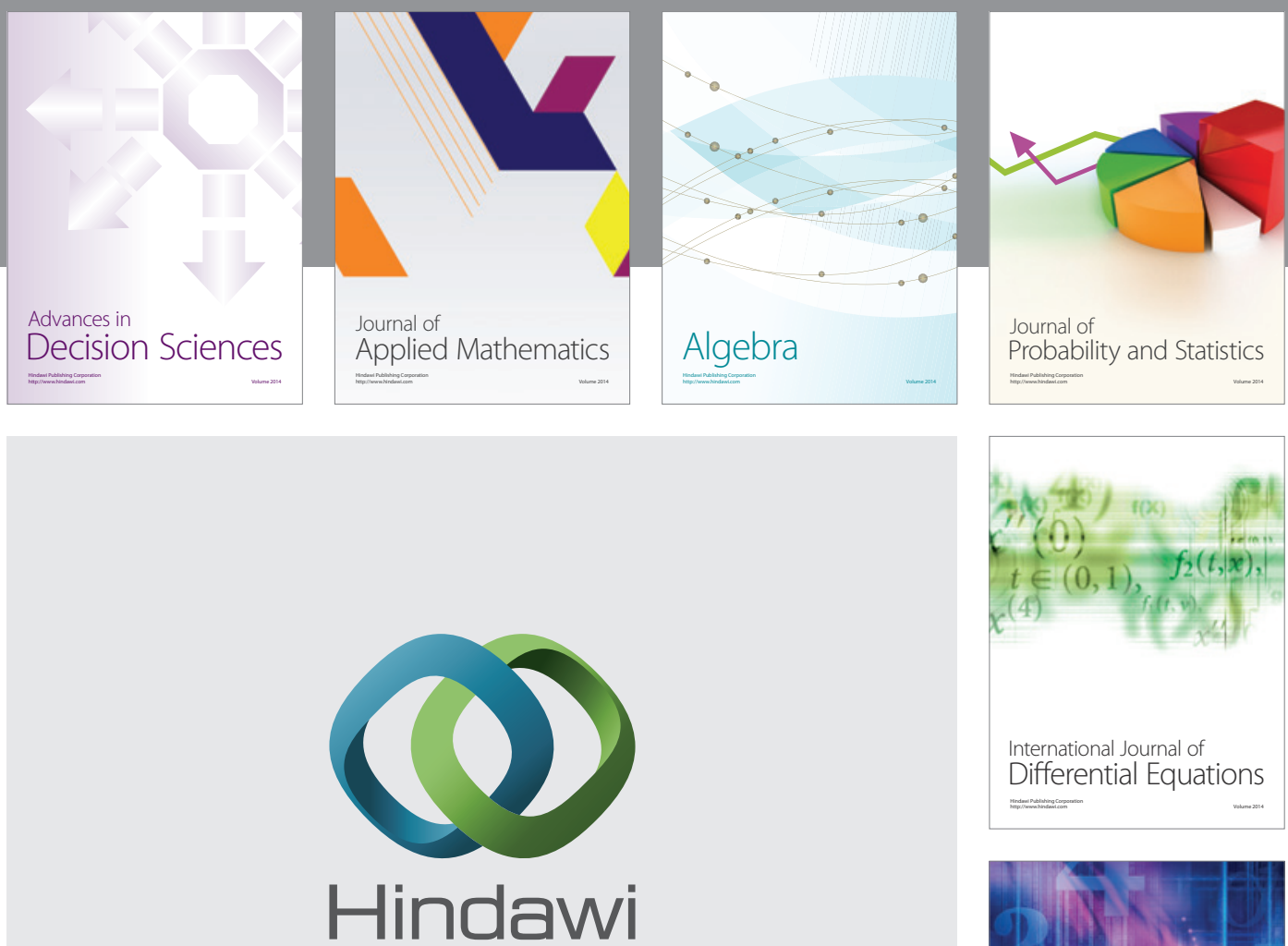

Submit your manuscripts at http://www.hindawi.com
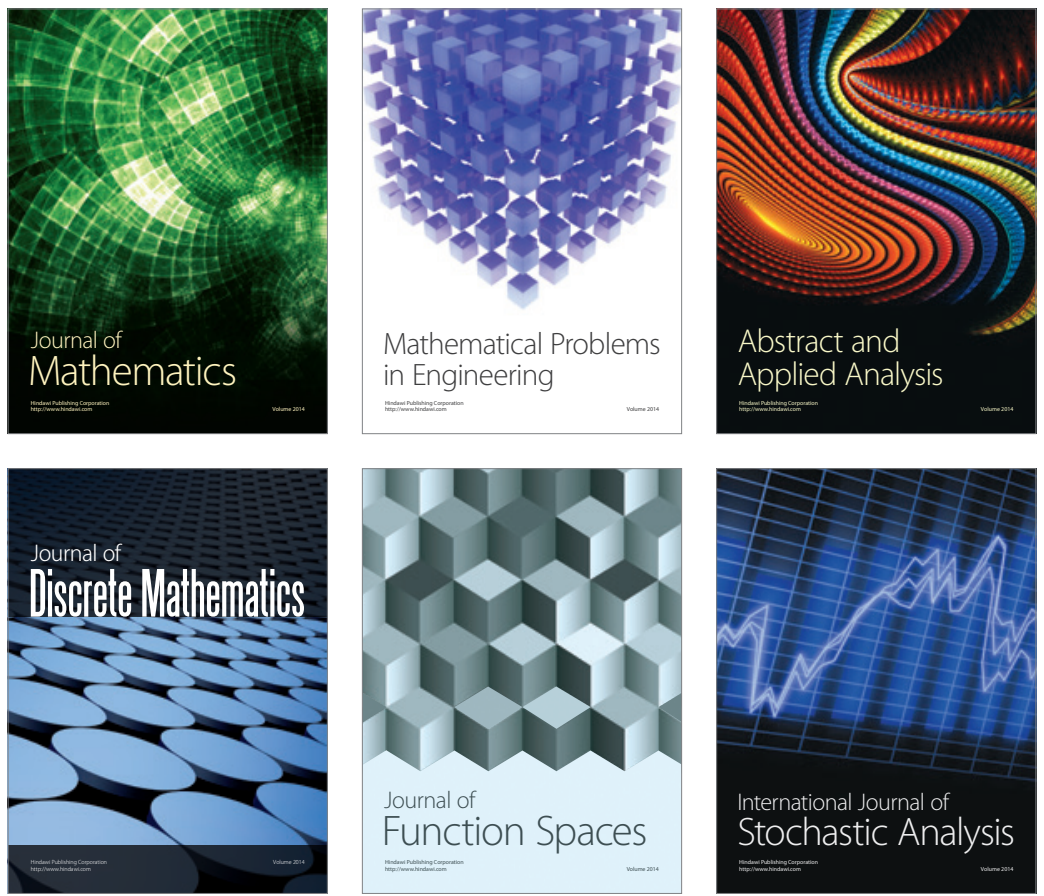

Journal of

Function Spaces

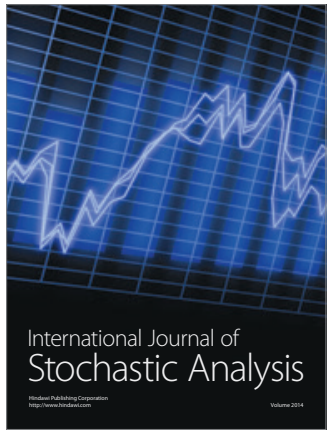

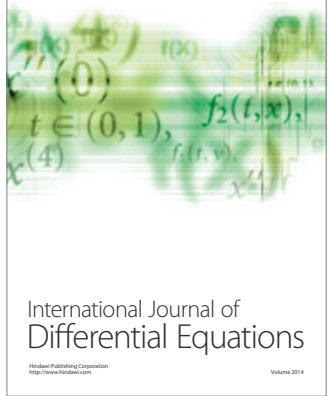
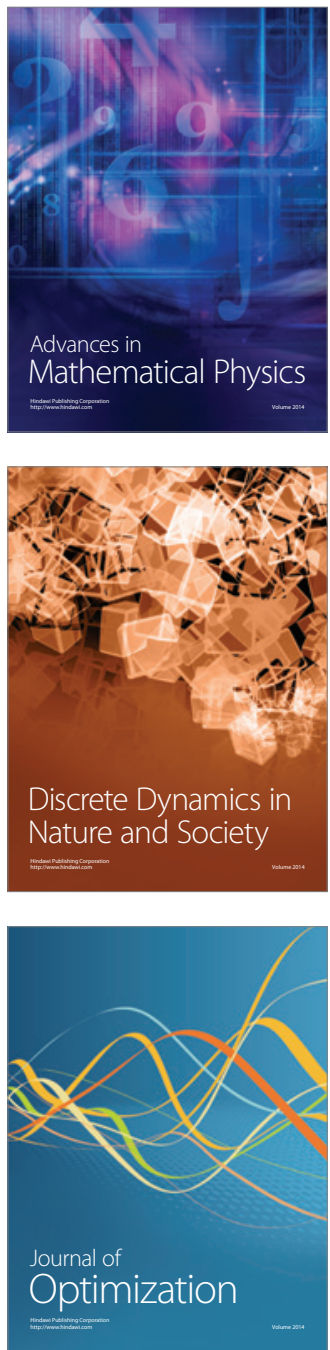